\title{
Débats publics et urbanité à Québec
}

\author{
Paul Villeneuve, Québec, Catherine Trudelle, Montréal, \\ Mathieu Pelletier, Québec, Florent Joerin, Québec
}

\section{Introduction}

La ville de Québec reste, encore aujourd'hui, proche de ses racines rurales (BÉlanger 1981). Contrairement à Montréal, peuplée en bonne part par l'immigration internationale, le peuplement de Québec repose, depuis la deuxième moitié du XIX ${ }^{\text {ème }}$ siècle, sur les campagnes environnantes (SAINT-HILAIRE 2001). Ceci alimente une forte continuité culturelle entre le rural et l'urbain au sein de la grande région de Québec. En conséquence, l'urbanité de Québec est de caractère récent, la pratique d'un genre de vie typiquement urbain se restreignant à une petite fraction du territoire. Deux arrondissements centraux, La Cité et Limoilou, forment un tissu urbain dense où $100^{\prime} 000$ habitants occupent $24 \mathrm{~km}^{2}$, alors que six autres arrondissements constituent un vaste espace suburbain où vivent 400 '000 personnes sur plus de $400 \mathrm{~km}^{2}$ (Figure $1)$.

Ce contraste éminemment tangible sous-tend un débat public continuel sur les vertus et les vices de la vie urbaine et de la vie suburbaine, de l'urbanité et de la «suburbanité». Le poids du nombre avantage cette dernière, mais la question environnementale sème le doute et soutient une certaine revalorisation de la première. Dans ce texte, il s'agira d'apprécier le rôle des débats publics dans la remise en question des modes de vie suburbains et dans la revalorisation de l'urbanité à Québec. Dans quelle mesure débats, controverses et conflits contribuent-ils à clarifier les enjeux associés à l'urbanité? Pourquoi, depuis un demi-siècle, ces débats sont-ils très médiatisés? Quelles sont les conséquences des multiples expériences d'institutionnalisation des débats qui ont pris forme dans la même période: un freinage des dynamismes de la société civile ou un élargissement des pratiques de gouvernance?

Nous nous appuyons sur le cas de Québec pour esquisser des réponses forcément partielles à ces questions. Après avoir précisé les notions de débat public et d'urbanité, nous caractérisons la reprise urbaine dans les quartiers centraux de la ville et montrons comment, dans le long terme, les débats publics ont joué un rôle central dans cette reprise. Nous terminons par une esquisse des formes de gouvernance urbaine qui émergent actuellement à Québec.

\section{Débats publics et urbanité: quels rapports?}

La notion d'urbanité peut prendre une forme purement descriptive, sans connotation positive ou négative. C'est ce que proposent certains spécialistes de la ville et de l'urbain. Ainsi, Lussault (2003: 950) définit l'urbain comme une organisation spatiale qui met en cause la densité et la diversité, l'urbanité désignant alors des situations où la densité et la diversité sont fortes. Il y a plus d'un demi-siècle, WIRTH (1938) caractérisait déjà la ville de la même façon, ajoutant toutefois la notion de taille à celles de densité et de diversité. Selon cette vision des choses, une agglomération urbaine de plus forte taille, de densité plus élevée et de diversité plus grande qu'une autre possède un coefficient d'urbanité plus grand. Notons également qu'au sein d'une même agglomération, les secteurs centraux connaissent généralement, mais pas nécessairement, une densité et une diversité plus marquées que celles des secteurs périphériques, comme le montrent les nombreuses études portant sur les gradients de densité (Bunting et al. 2002) et celles, moins nombreuses, qui abordent les dimensions de la diversité (VILLENEUVE 2009).

Dans son sens général, le mot urbanité est doté d'une connotation positive dont la dose varie selon quelques définitions glanées au hasard des dictionnaires: «ensemble des caractéristiques propres à la vie dans les villes»; «caractère fonctionnel et convivial de structures conçues et adaptées à la vie dans les villes»; «savoir-vivre empreint de raffinement» (ENCARTA 2009). Cette conception de l'urbanité comme «savoirvivre ensemble» se rapproche de celle élaborée par les chercheurs qui tiennent compte des émotions et des affects dans leur démarche (Bоснет 2008). Cette façon de penser l'urbanité se rapproche également de ce que l'UNESCO appelle l'humanisation des villes, dont les trois dimensions seraient le droit à la ville, l'établissement de formes participatives et le développement d'une solidarité urbaine active (UNESCO 1999). Ces dimensions reposent toutes sur un socle commun qu'on pourrait appeler la prise de parole citoyenne. Nous proposerons plus bas un indicateur qui tente d'intégrer les dimensions descriptives et normatives de l'urbanité.

À Québec, la prise de parole citoyenne se manifesta, entre autres, dans les débats entourant le réaménagement des quartiers centraux à compter du milieu des années 1960. Ce réaménagement accompagnait alors un processus d'expansion et de modernisation de l'État connu sous le nom de Révolution tran- 


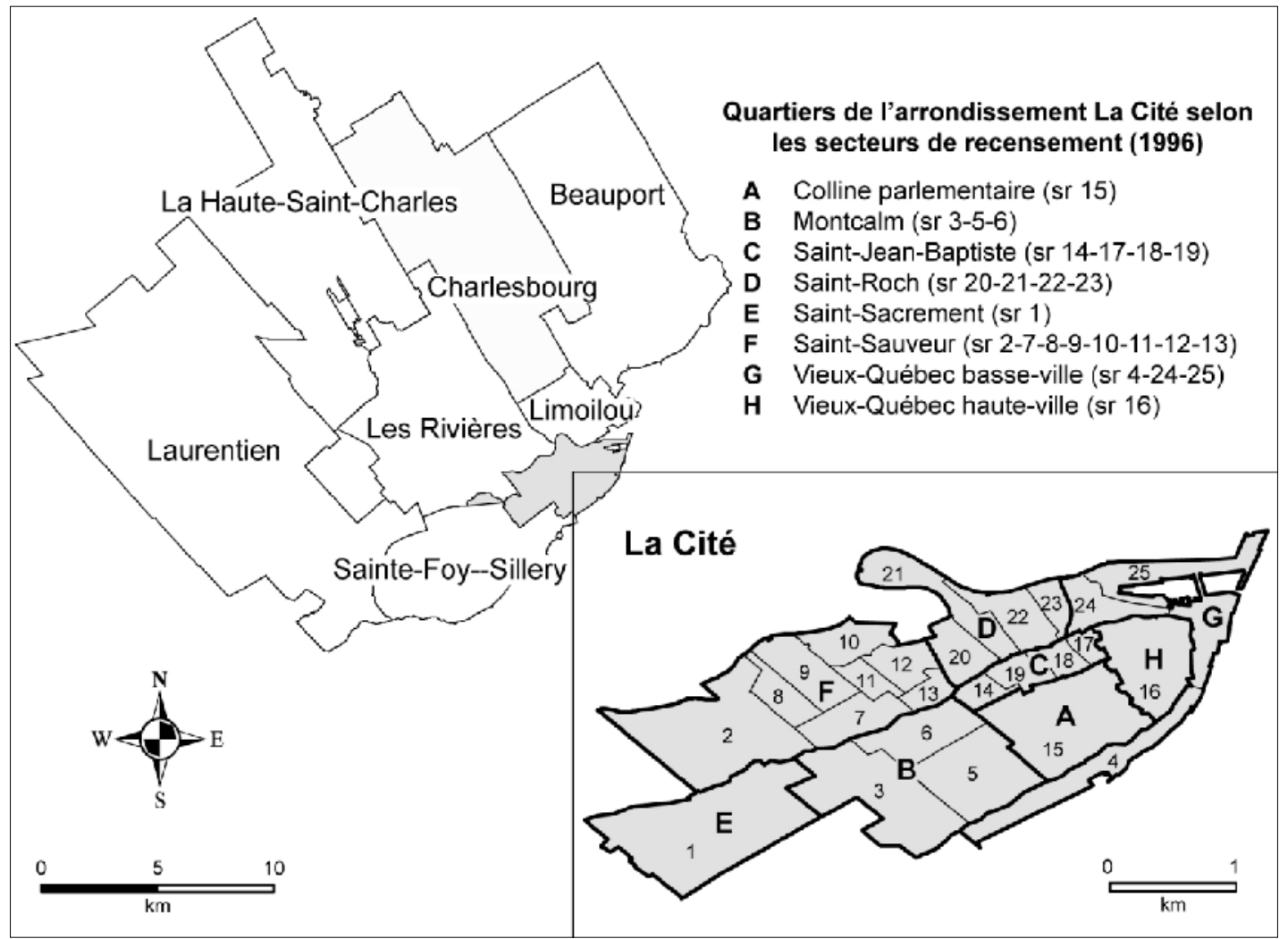

Fig. 1: Les arrondissements de la ville de Québec, les quartiers de l'arrondissement de La Cité et les secteurs de recensement qui les composent

Districts of Quebec City, neighbourhoods in the district of «La Cité» and census tracts of each neighbourhood Die Distrikte der Stadt Québec, die Quartiere des Distrikts La Cité und die Zensus-Gebiete der Quartiere Source: Statistique CANADA pour le découpage en secteurs de recensement et Ville DE Québec pour le découpage en quartiers et en arrondissements

quille (VILLENEuve 1981). La composante urbanistique de ce processus s'est traduite par un effort considérable de modernisation urbaine imposée conjointement par le gouvernement du Québec et l'administration de la ville. Des formes urbaines et suburbaines très nord-américaines sont alors apparues, la décennie des années 1970 ayant été particulièrement féconde à cet égard. Qu'il s'agisse des gratte-ciel de la Colline parlementaire (lieu du Parlement et du Gouvernement québécois), des grands centres commerciaux des banlieues, des autoroutes et de l'habitat pavillonnaire, Québec se modernisait alors sous la coupe d'un pouvoir provincial et municipal ignorant des pratiques de démocratie participative qui commençaient alors à se répandre en Europe et en Amérique.
Cette politique de modernisation entraîna la destruction d'une partie importante du stock de logement des quartiers centraux, provoquant en retour, dans ces quartiers, des oppositions populaires (HULBERT 1994). Ces oppositions ont pu, dès les années 1960 , s'inspirer d'un large mouvement social urbain (CASTELLS 1983). À Québec, comme dans plusieurs autres villes, les quartiers centraux devinrent rapidement des lieux d'expérimentation du débat urbain et de la citoyenneté, ce processus étant porté par un élargissement considérable de la scolarisation (à Québec entre 1951 et 2001, la proportion de personnes de plus de cinq ans ayant plus de treize années de scolarité passe de $10 \%$ à $50 \%$ ) menant à la recherche, par une fraction minoritaire mais non négligeable des nouvelles classes moyennes, d'un mode de vie urbain plutôt 
que suburbain. En 2001, lorsque des arrondissements furent découpés dans la ville élargie à la suite d'une restructuration municipale massive, on nomma l'arrondissement le plus central «La Cité» évoquant ainsi ce rôle d'agora où sont vigoureusement débattus les projets qui veulent concrétiser la nouvelle urbanité de Québec.

La parole aurait maintenant droit de cité dans cette ville de pouvoir. Mais quelle est son efficacité (BHERER 2006)? Les débats publics au sujet des projets urbains qui affectent les quartiers centraux sont-ils associés de façon mesurable à l'urbanité de ces quartiers? Pour répondre à cette question, nous proposons une démarche empirique simple. Il s'agit d'identifier et de mettre en rapport des indicateurs capables de nous aider à évaluer les covariations dans les niveaux d'urbanité et dans la vigueur des débats publics.

Pour ce qui est de l'urbanité, l'indicateur le plus évident est sans doute, tout simplement, l'importance de la reprise démographique à l'échelle des quartiers. Le choix de cet indicateur s'appuie sur une logique maintes fois évoquée en ce qui concerne l'attraction exercée par les diverses municipalités d'une agglomération: «les gens votent avec leurs pieds» (Tiebout 1956). En d'autres termes, les ménages cherchent à s'établir dans la municipalité ou le quartier qui répond le mieux à leurs désirs, compte tenu des contraintes qui s'exercent sur leur choix. Cet indicateur a l'avantage de subsumer certains aspects descriptifs et certaines connotations positives de la notion d'urbanité. D'une part, la croissance de la population dans un quartier dont la superficie ne change pas se traduit nécessairement par une augmentation de la densité (mais sans doute pas forcément par une plus grande diversité); d'autre part, cette croissance exprime un attrait pour la vie urbaine, la «gentrification comme désir» selon le mot de CAulfield (1988). À Québec, les huit quartiers qui composent l'arrondissement de La Cité ont connu une croissance de leur population entre 1996 et 2001 (voir l'axe des ordonnées des Figures 3 et 4), la plus faible étant celle du quartier Saint-Sauveur (1,07\%) et la plus forte celle du Vieux Québec basse-ville (12,31\%).

Pour ce qui est de l'ampleur des débats publics, elle peut être évaluée à l'aide de la couverture de presse accordée aux débats, controverses et conflits. Une base de données inédite a été constituée à cet effet à Québec (Pelletier et al. 2007; Trudelle 2005; VilleNEUVE et al. 2006). Plus de 2'000 événements conflictuels ont été relevés dans le principal quotidien local, Le Soleil, sur la période 1965-2000. De ces conflits, 482 sont localisés dans l'arrondissement de La Cité. Nous relevons la surface en $\mathrm{cm}^{2}$ consacrée par le journal à chaque conflit. Le recours à cette façon de mesurer les débats publics comporte des avantages et des inconvé- nients. Parmi les premiers, notons d'abord le caractère éminemment public de la source. Les journaux quotidiens nourrissent et sont nourris par «les» opinions publiques. Ils deviennent d'emblée des acteurs locaux de premier plan, ce qui invite à la prudence lorsqu'on les utilise comme source (GILBERT \& BRosseau 2002). Notons ensuite la granulométrie spatio-temporelle très fine associée à cette source ainsi que la possibilité de remonter dans le passé. Parmi les inconvénients, on trouve les biais de sélection et d'orientation véhiculés dans la presse. Une étude détaillée de ceux-ci (TruDELLE 2005) porte à conclure, que malgré ces imperfections, de nombreux chercheurs y ont recours car ils constatent que les avantages de cette source dépassent ses inconvénients.

\section{Reprise démographique au centre et débats publics}

Il s'agit maintenant de tester l'hypothèse voulant que la reprise démographique des quartiers centraux, où l'urbanité est généralement plus intense qu'en banlieue, soit associée à l'ampleur des débats portant sur les projets urbains destinés à ces quartiers. Certains de ces projets favorisent d'emblée la fonction résidentielle. D'autres portent sur des activités difficilement compatibles avec cette fonction quoique, dans la ville postindustrielle, cette compatibilité soit peut-être moins difficile à réaliser qu'à l'époque où les usines côtoyaient les résidences.

Des facteurs autres que les débats publics peuvent être associés à une reprise démographique dans les quartiers centraux. Déjà, Bourne (1992) avait identifié une telle reprise dans plusieurs villes canadiennes entre 1981 et 1986, et présenté un certain nombre de facteurs pouvant y être associés. Parmi ceux-ci, les facteurs suivants ont été observés à Québec: une certaine concentration au centre des emplois du tertiaire supérieur, ces emplois étant par ailleurs de plus en plus occupés par des femmes (SÉGuin \& Villeneuve 1987); une diminution de la taille des ménages (Rose \& VILLENEUVE 2006); la congestion qui fait augmenter la durée des navettes résidence-travail (BOUREL 2005); l'adoption du condominium comme moyen d'accès à la propriété (Сноко et al. 2006); la politique d'investissement des pouvoirs publics, elle-même liée aux débats publics (VILleneuve \& Trudelle 2008).

Par ailleurs, un facteur de nature économique n'a pas été étudié à Québec. Il s'agit de l'écart de rente foncière qui se creuse entre les quartiers centraux et périphériques pendant la période de dévalorisation des premiers, et qui diminuerait les coûts des réinvestissements au centre (par ailleurs risqués) de la part des acteurs privés et publics. Il s'agit d'un aspect de l'hypothèse du «rent gap» développée originellement 
par Sмiтh (1982). Nous nous inspirerons de cette hypothèse pour investiguer, à l'échelle des quartiers, l'effet d'une longue dévalorisation sur la reprise démographique et comparer le poids de ce facteur de nature économique à celui des débats publics, facteur éminemment politique. L'écart entre la rente foncière actuelle et la rente foncière potentielle étant difficile à calculer, nous aurons recours à un indice proposé par LEY (1986). Celui-ci mesure la valorisation/dévalorisation relative de chaque quartier par l'évolution du ratio du loyer moyen payé dans le quartier sur le loyer moyen de l'ensemble de l'agglomération. Nous avons calculé cet indice à chacun des recensements entre 1951 et 2006. Les quartiers ayant connu la plus profonde dévalorisation devraient aussi être ceux qui connaissent la meilleure reprise démographique.

Nous testons les hypothèses d'une relation entre les débats publics et l'écart de rente, d'une part, et la reprise démographique dans les quartiers centraux, d'autre part, en constituant trois séries chronologiques pour chacun des huit quartiers qui composent l'arrondissement de La Cité. Ces huit quartiers forment des regroupements de secteurs de recensement dont les limites n'ont pas bougé depuis 1941. Une première série est constituée par la population de chaque quartier à chacune des dates de recensement depuis 1941. Une deuxième série mesure le loyer moyen de chaque quartier par rapport à celui de l'agglomération à chacun des recensements. Enfin, une troisième série évalue l'évolution de l'ampleur des débats publics dans chaque quartier au moyen d'un indice d'activité conflictuelle per capita. Pour obtenir cet indice, la période de 1965 à 2000 est divisée en cinq phases de sept ans chacune. Pour chacune de ces phases et chacun des quartiers, nous divisons par la population du quartier la surface en centimètres carrés consacrée aux articles de presse portant sur les conflits. Ces trois séries chronologiques non linéaires sont généralisables à l'aide de fonctions polynomiales de degré deux pour chacun des huit quartiers de l'arrondissement de La Cité. Les courbes des divers quartiers ont sensiblement la même forme que celles obtenues pour le quartier de la Colline parlementaire (Figure 2):

$$
\begin{aligned}
& \text { Population }= \\
& \text { 3,0163·temps }{ }^{2}-557,960 \cdot \text { temps }+28971,00\left(\mathrm{R}^{2}=0,90\right) \\
& \text { Loyer }(\text { quartier/agglomération })= \\
& 0,0003 \cdot \text { temps }{ }^{2}-0,053 \cdot \text { temps }+3,21\left(\mathrm{R}^{2}=0,63\right) \\
& \text { Conflits }= \\
& -0,0095 \cdot \text { temps }^{2}+1,585 \cdot \text { temps }-63,45\left(\mathrm{R}^{2}=0,74\right)
\end{aligned}
$$

Les variations d'un quartier à l'autre portent sur les niveaux des courbes, sur les gradients d'augmentation et de diminution, ainsi que sur les points dans le temps où les maxima et les minima sont atteints. Par exemple, les niveaux des loyers relatifs sont plus élevés dans les quartiers de la haute-ville que dans ceux de la basseville; la déprise démographique est plus abrupte, et la reprise également, dans les quartiers les plus centraux (Vieux-Québec basse-ville, Vieux-Québec haute-ville et Saint-Roch); et dans ces quartiers, la période pendant laquelle l'activité conflictuelle est la plus forte est généralement plus tardive. On notera (Figure 2) que le point minimal de la courbe des loyers et le point maximal de la courbe des conflits sont antérieurs au point minimal de la courbe démographique. Nonobstant les variations d'un quartier à l'autre qui viennent d'être mentionnées, ce pattern est aussi obtenu pour les autres quartiers. Cette similitude de pattern autorise une analyse plus poussée des variations entre les quartiers. Si les débats publics sur la ville sont liés à l'urbanité, ceci devrait se refléter dans nos indicateurs. Plus spécifiquement, les quartiers où le niveau et, possiblement, la proximité dans le temps des débats publics sont élevés, devraient connaître une reprise démographique plus forte. De plus, les quartiers où les loyers relatifs ont atteint les niveaux les plus bas devraient aussi être ceux qui connaissent la meilleure reprise.

Pour tester ces relations, nous procédons à des analyses de régression sur les variations, parmi les huit quartiers de La Cité, dans les niveaux de loyers, d'activité conflictuelle et de reprise démographique (Figures 3 et 4). Le fait que les analyses ne portent que sur huit observations (quartiers) limite fortement le nombre de degrés de liberté. Pour ce qui est du lien entre le niveau des loyers relatifs et la reprise démographique, nous régressons le taux de croissance de la population entre 1996 et 2001 (période où la reprise est maximale) sur le niveau des loyers relatifs en 1981 (année où ce niveau est généralement le plus bas). La direction de la relation est conforme aux attentes, les quartiers ayant le loyer le plus faible en 1981 étant aussi ceux pour lesquels la reprise démographique est la plus forte, mais elle n'est pas significative au plan statistique, le niveau des loyers ne rendant compte que de $16,81 \%$ des variations dans la reprise démographique.

Pour analyser l'association entre l'activité conflictuelle et la reprise démographique (Figure 4), nous avons retenu le niveau d'activité per capita pendant la phase où ce niveau est le plus élevé. Nous avons aussi retenu le rang dans le temps $(1=1965-72, \ldots, 5=1993$ 2000) de la phase où il est maximal, ce dernier élément étant un indice de l'écart temporel entre des débats vigoureux et la reprise démographique. Par exemple, dans le cas du quartier de la Colline parlementaire, le niveau d'activité conflictuelle per capita pour la phase où il est maximal est de 3,21 (quartier «A», Figure 4) et cette phase est la quatrième (1986-1993). Compte tenu du faible nombre d'observations, la netteté des 


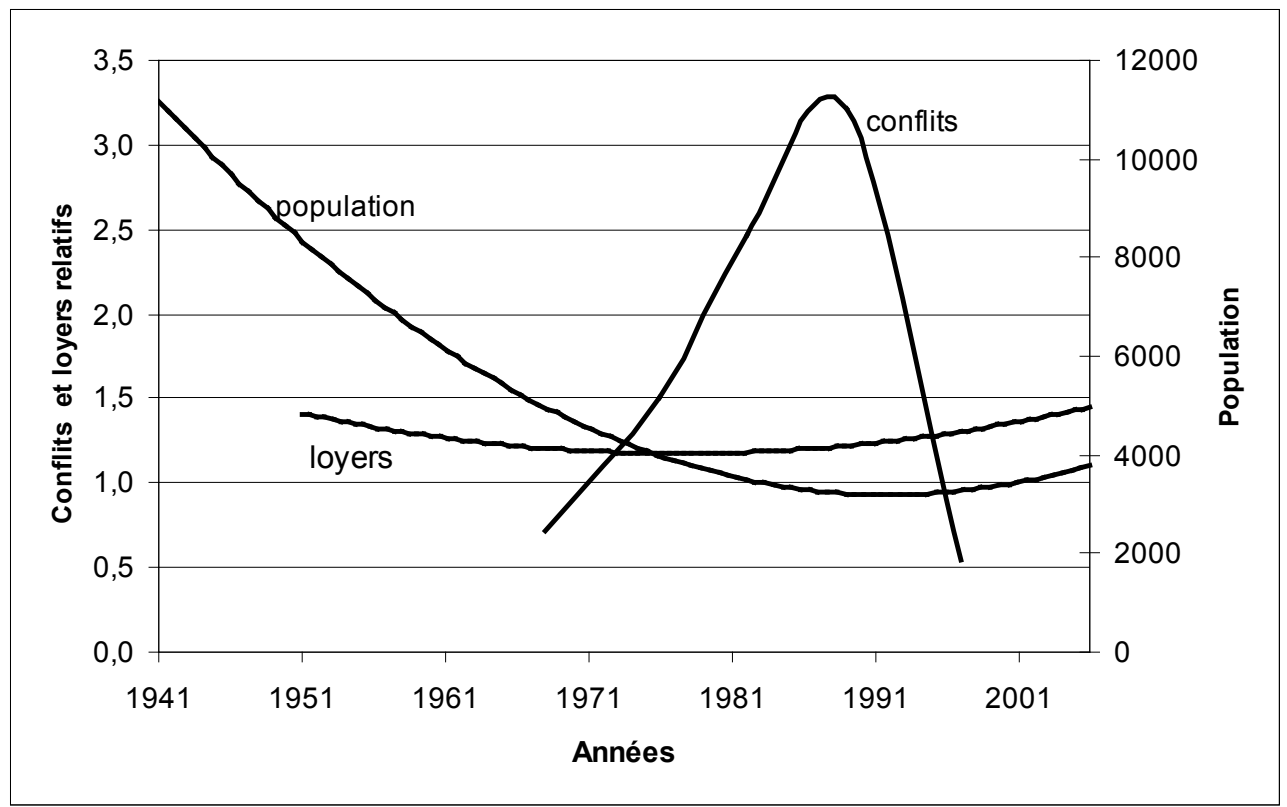

Fig. 2: Evolution de la population, des loyers et de l'ampleur des conflits, quartier de la Colline parlementaire, Québec, 1941-2001

Population development, level of rents and extent of conflict. The neighbourhood Parliamentary Hill, Québec City, 1941-2001

Bevölkerungsentwicklung, Mieten und Konflikt-Aktivität. Das Quartier Parliamentary Hill, Stadt Québec, 19412001

Source: Données sur la population et les loyers: Statistique Canada, recensements de la population. Données sur l'activité conflictuelle recueillies et analysées par les auteurs

relations obtenues est encourageante (Tableau 1 et Figure 4). L'activité conflictuelle maximale (ACM) et le rang de la période où se produit l'ACM sont tous deux associés de façon significative au taux de croissance de la population des quartiers entre 1996 et 2001. Conséquemment, nous ne pouvons rejeter l'hypothèse selon laquelle le niveau et la proximité dans le temps de l'activité conflictuelle sont associés positivement à la reprise démographique des quartiers centraux de Québec. Cette analyse devra éventuellement être bonifiée pour tenir compte des types de conflits qui devraient être différents selon qu'ils se produisent avant, pendant ou après la période de reprise démographique. Il reste que si l'on accepte la logique selon laquelle l'activité conflictuelle et le reprise démographique sont des indicateurs valables des débats publics et de l'urbanité, il en découle que de vigoureux débats publics auraient tendance à être associés à des gains d'urbanité. Mais que nous enseigne cet exercice d'analyse empirique sur l'évolution à plus long terme de l'urbanité de Québec?

\section{L'urbanité de Québec aujourd'hui: bilan et perspectives}

Nous avons proposé de considérer la reprise démographique des quartiers centraux comme un facteur de l'urbanité d'une ville. À cet égard, l'urbanité de Québec a progressé nettement entre 1996 et 2001 alors que la population de l'arrondissement de La Cité s'est accrue de plus de 2'000 personnes (de 60'015 à 62'110). Cet accroissement ne s'est toutefois pas poursuivi au même rythme par la suite. Entre 2001 et 2006, l'ajout démographique dans La Cité ne fut que de 550 personnes. Ces gains récents, s'ils sont mis en perspective, peuvent paraître faibles: en 1951, le territoire de La Cité comptait $116^{\prime} 000$ personnes. Ils sont toutefois socialement significatifs dans le contexte d'une suburbanisation agissant comme un rouleau compresseur depuis un demi-siècle: alors qu'en 1951, 40\% de la population de l'agglomération de Québec vivaient dans des milieux à caractère suburbain (c'est-à-dire en dehors des arrondissements actuels de La Cité et de 


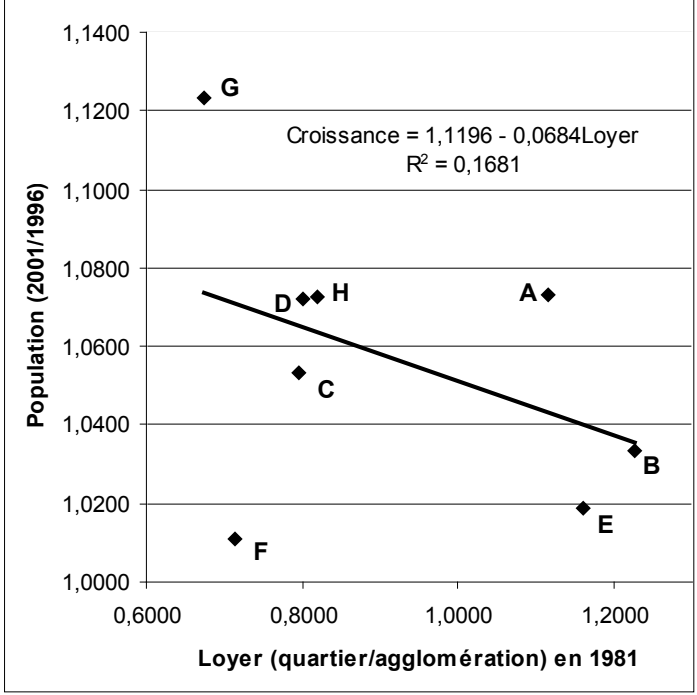

Fig. 3: Loyers relatifs et reprise démographique. Quartiers centraux de Québec

Relative rents and population growth. Inner city neighbourhoods, Quebec City

Relative Mieten und Bevölkerungswachstum. Innerstädtische Quartiere, Stadt Québec

Source: Statistique Canada, recensements de la population

Limoilou), en 2006 cette proportion atteignait 85\%. Dans ce contexte, choisir de «vivre en ville», c'est choisir d'être minoritaire.

Il est de plus possible que cette mise en minorité du genre de vie urbain soit affectée par la restructuration municipale récente qui s'est déroulée à Québec. En 2002, la douzaine de municipalités qui formaient la Communauté urbaine de Québec ont été fusionnées pour constituer une ville dont la population est passée de 169'000 à plus de 500'000 habitants. Avant la fusion, le genre de vie urbain était majoritaire au sein de la municipalité de Québec. Depuis la fusion, il est minoritaire, ce qui constitue un résultat non voulu par les citoyens des quartiers centraux qui étaient majoritairement en faveur de la fusion. Cette restructuration municipale peut avoir des répercussions qui restent à ce jour imprécises, car elles dépendent des attitudes qu'adopteront les citoyens et les élus «suburbains» à l'égard de leur ville. Considérons, par exemple, le projet de tramway qui fait présentement l'objet d'un débat public (Bourque 2007). Plusieurs reconnaissent que cet équipement contribuerait fortement à l'urbanité

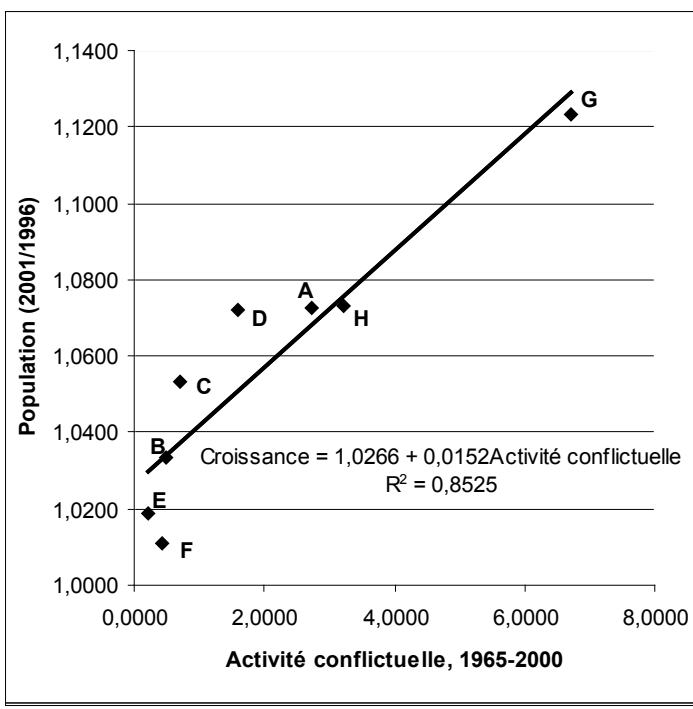

Fig.4: Activité conflictuelle et reprise démographique. Quartiers centraux de Québec

Conflict and population growth. Inner city neighbourhoods, Quebec City

Konflikt-Aktivität und Bevölkerungswachstum. Innerstädtische Quartiere, Québec Stadt

Source:Données sur la population:STATISTIQUe CANADA, recensements de la population. Données sur l'activité conflictuelle recueillies et analysées par les auteurs

de Québec, mais il s'agit d'un projet coûteux, dont on perçoit qu'il profitera d'abord aux résidents des quartiers centraux. Et ceci sera d'autant plus le cas si le mouvement d'opposition à toutes formes de densification des quartiers suburbains prend de l'ampleur (Moreault 2009).

Les dés sont cependant loin d'être jetés. Le chemin parcouru par la société locale depuis un demi-siècle montre un réel progrès au plan du «savoir vivre ensemble». Avant 1965, la ville de Québec était en proie au clientélisme et à l'esprit de clocher. Entre 1965 et 1977, efficacité et modernisation furent imposées d'en haut par l'élite d'affaires. Entre 1977 et 1989, les pratiques urbanistiques devinrent sensibles aux courants postmodernes s'exprimant dans la valorisation du patrimoine. Le Vieux-Québec fut inscrit au patrimoine mondial de l'UNESCO en 1985. De plus, les nouveaux édifices respectèrent mieux les gabarits et les styles existants. Toutefois, pendant cette période, la démocratie locale piétine.

De 1989 à 2001, le renouveau urbain prend de l'ampleur, impulsé par une série de démarches partici- 


\begin{tabular}{|c|c|c|c|}
\hline $\begin{array}{l}\text { Variable dépendante: taux de croissance de la population entre } \\
1996 \text { et } 2001\end{array}$ & B & $\boldsymbol{\beta}$ & $\mathbf{p}<$ \\
\hline \multicolumn{4}{|l|}{$\mathrm{R}^{2}$ ajusté $=0,914 ; \mathrm{N}=8$ quartiers } \\
\hline Constante & 0,998 & & 0,001 \\
\hline Activité conflictuelle maximale (ACM) & 0,013 & 0,772 & 0,002 \\
\hline Rang de la période où se produit l'ACM & 0,009 & 0,331 & 0,045 \\
\hline
\end{tabular}

Tab.1: Activité conflictuelle et reprise démographique dans les quartiers de l'arrondissement de La Cité, ville de Québec, 1965-2001: régression multiple. Les variables sont définies dans le texte. B = coefficient de régression; $\beta$ $=$ coefficient de régression standardisé; $\mathrm{p}<=$ probabilité que le coefficient soit égal à zéro.

Extent of conflict and population change in the neighbourhoods of the district La Cité, Quebec City, 1965-2001: multiple regression. Variables are defined in the text. $B=$ regression coefficient; $\beta=$ standardized regression coefficient; $p<=$ probability that coefficient is equal to zero.

Konflikt-Aktivität und demographischer Wandel in den Quartieren des La Cité Distrikts, Stadt Québec, 19652001: multiple Regression. Die Variablen werden im Text definiert. $B=$ Regressionskoeffizient; $\beta=$ standardisierter Regressionskoeffizient; $p<=$ Wahrscheinlichkeit, dass der Koeffizient gleich Null ist.

Source: Données de population: Statistique CANada, recensements de la population. Données sur l'activité conflictuelle recueillies et analysées par les auteurs

patives (BHERer 2003), à l'instar des processus à l'œuvre dans d'autres villes, à Lausanne par exemple (RACine 2008). Parmi ces démarches citons la création d'un Bureau des consultations publiques, la tenue fréquente d'audiences publiques, les Commissions consultatives permanentes et ad hoc et, surtout, les conseils de quartier (BHERER 2006). Entre 1990 et 1996, il y a institutionnalisation de la norme participative et baisse du niveau de conflits (Figure 2). Après 2001 et la restructuration, les institutions participatives sont implantées dans la ville élargie. Depuis 2005, les administrations qui se sont succédées à Québec se sont généralement conformées à la politique de consultation publique mise en place depuis 1989 .

Il reste à voir comment cette politique pourra cohabiter, à plus long terme, avec le régime de gouvernance en train d'être instauré par l'administration actuelle depuis l'automne 2007. Ce régime met en œuvre une gouvernance urbaine par projets que Pinson (2006: 62) décrit comme suit:

«Les projets sont des dispositifs d'action collective trans-sectoriels, partenariaux et mobilisant sur des temps relativement longs une pluralité d'acteurs, de groupes et d'institutions. Ils ouvrent la fabrique des politiques urbaines au-delà de la communauté restreinte des élus et des planificateurs publics et font interagir des élites urbaines autour de la définition du futur des villes».

Ce régime de gouvernance où les élites dominent pourrait éventuellement remettre en question le long et difficile processus de démocratisation urbaine vécu à Québec depuis quelques décennies.

\section{Bibliographie}

BÉLANGER, M. (1981): L'urbanité de Québec. - In: Cahiers de géographie du Québec 25, 64: 11-16.

BHERER, L. (2003): Une lecture institutionnaliste du phénomène participatif: la politique consultative de la Ville de Québec. - Thèse de doctorat, Institut d'études politiques de Bordeaux.

BHERER, L. (2006): Le cheminement du projet de conseils de quartier à Québec (1965-2006): un outil pour contrer l'apolitisme municipal? - In: Politique et Sociétés 25, 1:31-56.

Bochet, B. (2008): Les affects au cœur des préoccupations urbaines et urbanistiques: la réintroduction du sensible pour penser et concevoir la ville. - In: Geographica Helvetica 63, 4: 253-260.

Bourel, C. (2005): Les impacts du Métrobus de Québec. La question du report modal. - Québec: Université Laval, École supérieure d'aménagement du territoire et de développement régional, mémoire de deuxième cycle.

Bourne, L. (1992): Population turnaround in the Canadian inner city: Contextual factors and social consequences. - In: Canadian Journal of Urban Research 1, 1: 66-89.

Bourque, F. (2007): Un projet génial mais pas nécessaire. - In: Quotidien Le Soleil, 15 septembre: 8.

Bunting, T., Filion, P. \& H. Prinston (2002): Density gradients in Canadian metropolitan regions, 19711996: Differential patterns of central area and suburban growth and change. - In: Urban Studies 39, 13: 2531-2552.

CAstells, M. (1983): The city and the grassroots. A cross-cultural theory of urban social movements. - Berkeley: University of California Press. 
CAulfield, J. (1988): Gentrification and desire. - In: Canadian Review of Sociology/Revue canadienne de sociologie 26, 4: 617-632.

Сhoкo, M., Sénécal, G. \& N. Vachon (2006): La copropriété divise dans la région métropolitaine de Montréal et au Québec. - Montréal: Institut national de la recherche scientifique, Urbanisation, Culture et Société.

ENCARTA (2009): Dictionnaire Encarta (C) 20022008 Microsoft Corporation. - http://fr.ca.encarta. $\mathrm{msn}$.com/dictionary_2016036554/urbaniteC3A9.html 10.05.2009.

GILBERT, A. \& M. Brosseau (2002): Le journal, acteur urbain? Le Droit et la vocation du centre-ville de Hull. - In: Recherches sociographiques 43, 3: 517-548.

Hulbert, F. (1994): Essai de géopolitique urbaine et régionale. La comédie urbaine de Québec. - Laval: Éditions du Méridien.

LEY, D. (1986): Alternative explanations for inner-city gentrification: A Canadian assessment. - In: Annals of the Association of American Geographers 76, 4: 521535.

Lussault, M. (2003): Urbanité. - In: LÉvy, J. \& M. LusSAULT (éds): Dictionnaire de la géographie et de l'espace des sociétés. - Paris: Belin: 966-967.

Moreault, É. (2009): Densifier, mais pas à Sainte-FoySillery... - In: Quotidien Le Soleil, 16 février: 19.

Pelletier, M., Joerin, F. \& P. Villeneuve (2007): La décision territoriale en conflit. Ville de Québec, 1989 à 2000. Essai de modélisation spatiale. - Environnement urbain/Urban Environment 1: 76-94. http://www.vrm. ca/EUUE/vol1_2007/M-2007-13.pdf 16.05.2009.

Pinson, G. (2006): Projets de ville et gouvernance urbaine. Pluralisation des espaces politiques et recomposition d'une capacité d'action collective dans les villes européennes. - In: Revue française de science politique $56,4: 619-651$.

RACINE, J.-B. (2008): Projet urbain et démarche participative: contribution géographique à la possibilité de choisir sa ville aujourd'hui, quelques leçons de l'expérience lausannoise. - In: Bulletin de la Société géographique de Liège 50:5-15.

Rose, D. \& P. Villeneuve (2006): Life stages, living arrangements and lifestyles. - In: Bunting, T. \& P. FiLIon (éds): Canadian cities in transition. - Toronto: Oxford University Press: 138-153.

SaInT-Hilaire, M. (2001): Entre hier et demain. - In: Courville, S. \& R. Garon (éds): Québec, ville et capitale. - Sainte-Foy: Presses de l'Université Laval:330-333.

SÉGuin, A.-M. \& P. VIllLeneuve (1987): Du rapport hommes-femmes au centre de la Haute-Ville de Québec. - In: Cahiers de géographie du Québec 31, 83: 189-204.

SмIтH, N. (1982): Gentrification and uneven development. - In: Economic Geography 58, 2: 139-155.

Tiebout, C. (1956): A pure theory of local expenditures. - In: The Journal of Political Economy 64, 5: 416-424.

Trudelle, C. (2005): Visibilité de la participation des femmes aux conflits urbains à Québec entre 1965 et 2000. - Thèse de doctorat, Université Laval, École supérieure d'aménagement du territoire et de développement régional.

UNESCO (1999): Cité, citoyen, civilisation. - http:// www.unesco.org/courier/1999_06/fr/edito/txt1.htm 12.05.2009.

Villeneuve, P. (1981): La ville de Québec comme lieu de continuité. - In: Cahiers de géographie du Québec 25, 64: 49-60.

Villeneuve, P. (2009): Mesurer la diversité et les inégalités dans les villes canadiennes. - In: SÉNÉCAL, G. \& L. BHERER (éds): La métropolisation et ses territoires. - Québec: Presses de l'Université du Québec: 253268.

Villeneuve, P., Trudelle, C., Pelletier, M. \& M. ThÉRIAUlt (2006): Acteurs urbains en conflit, Québec, 1965-2000: essai d'analyse statistique. - In: Géocarrefour 81, 2: 135-141.

Villeneuve, P. \& C. Trudelle (2008): Retour au centre à Québec: la renaissance de La Cité est-elle durable? - In: Recherches sociographiques 49, 1:25-45.

WIRTH, L. (1938): Urbanism as a way of life. - In: American Journal of Sociology 91, 1:1-24.

\section{Résumé: Débats publics et urbanité à Québec}

La suburbanisation de Québec s'est déroulée à un rythme rapide au cours des cinquante dernières années, ce qui n'a pas manqué d'entraîner des débats de plus en plus amples sur les avantages et les inconvénients des genres de vie urbains et suburbains. Les quartiers centraux, après avoir perdu près de la moitié de leur population entre 1950 et 1990, ont connu une reprise démographique, particulièrement vive entre 1996 et 2001. Cette reprise, vue ici comme une forme de revalorisation des genres de vie urbains, semble associée assez étroitement avec la vigueur des débats publics portant sur des enjeux de développement à l'échelle des quartiers. Ce processus politique jouerait même un plus grand rôle dans cette revalorisation que les mécanismes économiques généralement associés à l'hypothèse du «rent gap». Toutefois, les effets de démocratisation locale associés à ce processus pourraient être remis en question par le régime élitiste de gouvernance par projets en train de s'implanter dans la ville.

Mots-clés: débats publics, urbanité, quartiers centraux, ville de Québec, reprise démographique

\section{Zusammenfassung: Öffentliche Debatten und Urbanität in Québec}

Während der letzten fünfzig Jahre verlief die Suburbanisierung in Québec rapid. Dies hat zunehmend öffentliche Debatten über die Vor- und Nachteile städtischer gegenüber suburbanen Lebensweisen 
ausgelöst. Innerstädtische Quartiere weisen zwischen 1996 und 2001 ein signifikantes demographisches Wachstum auf, nachdem sie zwischen 1950 und 1990 fast die Hälfte ihrer Einwohner verloren hatten. Diese Zunahme könnte als eine Wiederaufwertung städtischer Lebensstile bei einem Teil der Bevölkerung interpretiert werden, ein Prozess, der näher verbunden zu sein scheint mit der Prägnanz öffentlicher Debatten als mit der Hypothese des «rent gap». Allerdings scheinen die Demokratisierungseffekte, die diese zunehmenden Debatten begleitet haben, gefährdet durch das elitäre Regime der Governance, wie es sich derzeit darstellt.

Schlüsselwörter: Öffentliche Debatten, Urbanität, zentrale Quartiere, Stadt Québec, demographische Kehrtwende

\footnotetext{
Abstract: Public debates and urbanism in Quebec

City

During the last fifty years, suburbanisation in Quebec City has been rapid. This has triggered increasingly intensive public debates about the relative advantages of urban versus suburban ways of living. Inner city neighbourhoods witnessed a significant demographic increase between 1996 and 2001, after having lost almost half of their population between 1950 and 1990. This gain may be interpreted as a revalorisation of urban ways of living among a part of the population, a process which seems more closely associated with the impact of public debates than with the «rent gap» hypothesis. However, the democratisation effects which have accompanied mounting public debates may be endangered by the elitist regime of governance now being put into place.
}

Keywords: public debates, urbanism, inner city neighbourhoods, Quebec City, population turnaround

Prof. Dr. Paul Villeneuve, Centre de recherche en aménagement et développement (CRAD), Pavillon Félix-Antoine-Savard, Local 1612, Université Laval, Québec (Québec), Canada, G1V 0A6.

e-mail: paul.villeneuve@crad.ulaval.ca

Prof. Dr. Catherine Trudelle, Chaire de recherche du Canada sur les conflits socioterritoriaux et la gouvernance locale, Département de géographie, Université du Québec à Montréal, Case postale 8888, Succursale Centre-Ville, Montréal (Québec), H3C 3P8.

e-mail: trudelle.catherine@uqam.ca

Dr. Mathieu Pelletier, Chaire de recherche du Canada sur les conflits socioterritoriaux et la gouvernance locale, Département de géographie, Université du Québec à Montréal, Case postale 8888, Succursale Centre-Ville, Montréal (Québec), H3C 3P8.

e-mail: mathieu.pelletier@crad.ulaval.ca

Prof. Dr. Florent Joerin, Chaire de recherche du Canada en Aide à la décision territoriale, Pavillon Félix-Antoine-Savard, Local 1614, Université Laval, Québec (Québec), Canada, G1V 0A6.

e-mail: florent.joerin@crad.ulaval.ca

Manuskripteingang/received/manuscrit entré le 5.5.2009

Annahme zum Druck/accepted for publication/accepté pour l'impression: 21.12.2009 